\title{
Frecuencia del manejo quirúrgico con malla de la Incontinencia Urinaria de Esfuerzo en el Hospital Nacional Arzobispo Loayza
}

Alicia Marleny Valderrama Palomino', Diego Iván Valdivia Estrada', María Francesca Valdivia Francia', Carlos Francisco Vargas López', Mijail Enrique Vargas Torrejón', Diana Lucy Vásquez Agurto', Alex Alberto Guibovich Mesinas²

\section{RESUMEN}

Objetivo: Determinar la frecuencia del manejo quirúrgico con malla de la incontinencia urinaria de esfuerzo en el HNAL durante el año 2012.

Material y Metodos: estudio observacional, descriptivo y retrospectivo. La población incluyó todas las muj eres diagnosticadas con IUE y que recibieron tratamiento quirúrgico en el Servicio de Ginecología del HN A. Loayza con registro operatorio completo en el libro de Sala de Operaciones. Se llenó una ficha de recolección de datos, se creó una base de datos y se realizó el análisis con el programa estadístico Excel.

Resultados: 60 pacientes fueron sometidas a cirugía por IUE, con o sin distopia genital agregada y con patologías subyacentes. El promedio de edad de las pacientes fue de 59 años. El uso de mallas fue del 73,33\% de los procedimientos realizados. Conclusiones: La técnicas de suspensión uretral sin tensión con abordaje por vía vaginal fue la de mayor uso en el Hospital Loayza, con la ventaj a que se puede combinar con otras cirugías del tracto genital como histerectomía abdominal o vaginal y cura quirúrgica de cistocele. (Horiz Med 2014; 14(1): 38-41)

Palabras clave: incontinencia urinaria, cirugía con malla (Fuente: DeCS BIREME).

Frequency Mesh Surgical Management of Urinary Incontinence in the Arzobispo Loayza National Hospital

\section{ABSTRACT}

Objective: Determine the frequency of Mesh Surgical Management of Urinary Incontinence in the HNAL during 2012. Material and Methods: observational, descriptive and retrospective study. The population includes all women diagnosed with SUI and underwent surgery in the Department of Gynecology A. Loayza NH full operative registration book Operating Room. A form of data collection was filled, created a database and statistical analysis with the Excel program was performed. Results: 60 patients underwent surgery for SUI with or without genital dystopia aggregate and underlying pathologies. The average patient age was 59 years. The use of Mesh was $73.33 \%$ of the procedures performed.

Conclusions: The techniques tension-free urethral suspension vaginal approach was the most used in the Loayza Hospital, with the advantage that can be combined with other genital tract surgeries like abdominal or vaginal hysterectomy and surgical cure of cystocele. (Horiz Med 2014; 14(1): 38-41)

Keys words: mesh surgical, urinary incontinence (Source: MeSH NLM).

1 Alumnos de Sexto año de la FMH-USMP

2 Profesor de Ginecología y Obstetricia de la FMH-USMP 


\section{INTRODUCCIÓN}

La incontinencia urinaria de esfuerzo (IUE) es un problema de salud importante para la población general y aunque no afecta en forma directa la mortalidad, sí es una patología que genera gran compromiso de la calidad de vida de los pacientes afectados. Ocurre en 15 a $30 \%$ de las mujeres después de los 30 años, por lo que es una causa frecuente de consulta ginecológica (1).

En la IUE, los músculos pélvicos del esfínter, que sostienen la vejiga y uretra, están debilitados. El esfínter no puede impedir el flujo de orina cuando se ejerce presión sobre el abdomen (como cuando uno tose, se ríe o levanta algo pesado) $(2,3)$.

Puede presentarse por debilitamiento de los múscul os pélvicos que sostienen la vejiga y la uretra o debido a que el esfínter uretral no está funcionando correctamente. El debilitamiento puede ser causado por: parto, lesión en el área uretral, medicamentos o cirugía del área pélvica $(4,5)$.

La IUE, es el tipo más común de incontinencia urinaria en las mujeres (6).

Esta patología, genera un dilema en su manejo, ya que se han probado distintas técnicas quirúrgicas tanto por vía abdominal como vaginal con variados resultados. Las de mejor resultado a corto y largo plazo han sido la colpo-suspensión de Burch por vía abdominal y la malla vaginal libre de tensión (TVT) por vía vaginal.Según un estudio realizado en el Hospital Fuenlabrada de Madrid-España, la corrección de la IUE mediante mallas libres de tensión en régimen de cirugía mayor ambulatoria (CMA) es una opción viable que supone un ahorro substancial de recursos. Este tratamiento presenta un índice de curación del $92 \%(1,2)$. Además, la incidencia de complicaciones con las técnicas de malla vaginal libre de tensión es baja $(3,7)$.

Debido a la alta incidencia de mujeres adultas mayores con IUE y al avance del manejo quirúrgico de la misma con el uso de malla, el objetivo de este trabajo es determinar la frecuencia del manejo quirúrgico con malla de la incontinencia urinaria de esfuerzo en el HNAL durante el año 2012.

\section{MATERIAL Y MÉTODOS}

Estudio observacional, descriptivo y retrospectivo. La población incluyótodaslasmuj eresdiagnosticadas con IUE y que recibieron tratamiento quirúrgico en el Servicio de Ginecología del Hospital Nacional Arzobispo Loayza, durante el 2012 que tuvieron su historia clínica completa.

La recolección de datos de los pacientes operados por IUE se realizó mediante la revisión del libro de Registro de la Sala de Operaciones del Servicio de Ginecología.

La información recogida fue ingresada en una ficha de recolección de datos específica que se elaboró. El procesamiento y análisis estadístico de los datos se realizó en el programa Excel.

\section{RESULTADOS}

60 pacientes fueron sometidas a cirugía por IUE, con o sin distopia genital agregada y con patologías subyacentes. El promedio de edad de las pacientes operadas de esta patología fue de 59 años.

En cuanto al diagnóstico preoperatorio, todas las pacientes $(60$ mujeres) presentaron IUE. Sólo dos de ellas, acudieron exclusivamente con ese problema a consultorio. En 53 pacientes, se asoció el diagnóstico de distopia genital (DG), cuatro pacientes ingresaron con el diagnóstico de tumoración pélvico - abdominal (TPA) por miomatosis uterina, cinco pacientes acudieron con hemorragia uterina anormal (HUA) y una con endometriosis. Tabla 1.

Tabla 1. Diagnóstico preoperatorio

\begin{tabular}{|cc|}
\hline DIAGNÓSTICO & NÚMERO DE \\
\hline PREOPERATORIO & PACIENTES \\
\hline IUE & 60 \\
\hline Distopia Genital & 53 \\
Miomatosis & 4 \\
HUA & 5 \\
\hline Endometriosis & 1 \\
\hline Obesidad (tipo 1) & 2 \\
Sobrepeso & 1 \\
\hline
\end{tabular}


La mayoría presentó DG de III y IV Grado (36 pacientes) constituyendo el $60 \%$. Ninguna presentó Grado I. Tabla 2.

Tabla 2. Grado de distopia genital.

\begin{tabular}{cc|}
$\begin{array}{c}\text { GRADO DE } \\
\text { DISTOPIA GENITAL }\end{array}$ & $\begin{array}{c}\text { NÚMERO DE } \\
\text { PACIENTES }\end{array}$ \\
\hline I & 0 \\
II & 17 \\
III & 26 \\
IV & 10 \\
\hline
\end{tabular}

Con respecto a la hemorragia uterina anormal, 5 pacientes tuvieron dicho antecedente. Cuatro de ellas (80\%), presentaron patología tumoral (pólipo y mioma submucoso). La otra paciente tuvo hiperplasia endometrial benigna. Tabla 3.

Tabla 3. HUA

\begin{tabular}{cc}
\hline TIPOS DE HUA & $\begin{array}{c}\text { NÚMERO DE } \\
\text { PACIENTES }\end{array}$ \\
\hline Pólipos & 2 \\
\hline Miomatosis & 2 \\
\hline Posmenopausica & 1 \\
\hline
\end{tabular}

En cuanto a los procedimientos realizados, la Histerectomía vaginal con colporrafia anteroposterior mas la malla TOT con $47 \%$, fue la que predominó. Seguida por la colporrafia anterior más TOT, solo malla TOT e histerectomía vaginal más colporrafia anterior más TOT con $5 \%$ cada uno.

\section{DISCUSIÓN}

Se han descrito una gran variedad de técnicas quirúrgicas en el tratamiento de la incontinencia urinaria (8). En 1995 se produjo una revolución en el tratamiento de la IUE cuando se introdujo la TVT (9).

Buscando alternativas surgió la técnica de la TOT (10). La que tenía como principales ventajas: la posición más anatómica de la cinta, la ausencia de incisiones abdominales, la disminución del riesgo de lesión vesical e intestinal al no pasar la aguja por el espacio retropúbico y, que no requiere cistoscopia $(8,10)$.

En el presente estudio, encontramos que el promedio de edad de las pacientes operadas de IUE fue de 59 años, edad que concuerda con algunos estudios $(11,12)$ donde mencionan que la IUE es muy prevalente en las mujeres de edad avanzada y supone también la forma más común de IU en las mujeres menores de 75 años, ya que afecta a casi un 50\% de ellas.

Hallamos que el tratamiento mayormente utilizado fue el quirúrgico y dentro de este la técnica del TOT con un $73.33 \%$ algo que concuerda con la literatura donde encontramos que las técnicas de suspensión uretral sin tensión (técnicas mínimamente invasivas de tipo TVT o TOT) se han convertido en las pruebas de referencia y han desplazado a las técnicas de colposuspensión como la de Burch, que durante años fue la técnica más utilizada (13-15).

En conclusión, las técnicas de suspensión uretral sin tensión con abordaje por vía vaginal son las técnicas de mayor uso hoy en día, en hospitales de alta complejidad, como el Hospital Loayza.

\section{Fuentes de financiamiento}

Los autores han autofinanciado el presente artículo.

\section{Conflictos de interés}

Los autores declaran no tener ningún conflicto de interés.

\section{REFERENCIAS BIBLIOGRÁFICAS}

1. Abrams P, Blaivas JG, Stanton SL, Andersen JT. The International Continence Society Committee on Standardization of Terminology: the standardization of terminology of lower urinary tract function. Scand J Urol Nephrol 1988;114 (suppl):5-19.

2. Abrams $P$, Cardozo $L$, Fall $M$, et al. The standardisation of terminology of lower urinary tract function. Neurourol Urodyn 2002;21:167-178. 
3. Barentsen $\mathrm{R}$, et al. Continuous low dose estradiol released from a vaginal ring versus estriol vaginal cream for urogenital atrophy. Eur J Obstet Gynecol Reprod Biol 1997;71(1):73-80.

4. C. Hampel, y col. Revisión de epidemiología de la incontinencia urinaria.Urology1997; 50 (64): 4-14.

5. Cardozo L, et al. Meta-analysis of estrogen therapy in the management of urogenital atrophy in postmenopausal women: second report of the Hormones and Urogenital Therapy Committee. Obstet Gynecol 1998;92(4 Pt 2):722-7.

6. Chompootaweep S, et al. The use of two estrogen preparations (a combined contraceptive pill versus conjugated estrogen cream) intravaginally to treat urogenital symptoms in postmenopausal Thai women: a comparative study. Clin Pharmacol Ther 1998;64(2):204-10.

7. Zapardiel I, de la Fuente-Valero J, Botija J, Pérez-Medina T, Díez M, Bajo JM. Valoración de la eficacia de las mallas libres de tensión y satisfacción de 241 pacientes en la corrección de la incontinencia urinaria. Actas Urol Esp 2008;32:637-641.

8. Veloso D, Saens $P$, Ainardi $M$, Olivares $A$, et al. Cinta suburetral transobturatriz libre de tensión para el tratamiento de la incontinencia de orina de esfuerzo: 3 años de seguimiento. Rev. chil obstet. ginecol. [en Internet]. 2010 [citado 2012 Mar 20]; 75(4): 240-246. Disponible en: http://www.scielo cl/scielo.php?script=sci_ arttext\&pid $=\quad$ S0717-75262010000400005\&lng $=$ es

9. Kang Myung B, Kim Hyeong G, Paick Sung H, Lho Yong $\mathrm{S}$, Park Hyoung $\mathrm{K}$. Intraoperative maximal urethral closing pressure measurement: a new technique of tape tension adjustment in transobturator sling surgery?. Int. braz j urol. [on Internet]. 2011 Dec [cited 2012 May 16] ; 37(6): 751-757. Available from:http: / /www.scielo.br/scielo.php?script $=$ sci_ arttext\&pid=S1677-55382011000600011\&lng=en
10. Solà Dalenz V., Pardo Schanz J., Ricci Arriola P., Guiloff Fische E., Chiang Miranda H. Cirugía mínimamente invasivaenincontinenciaurinariafemenina:TVT-O.Actas Urol Esp [en Internet]. 2006 Ene [citado 2012 Mar 22]; 30(1):61-66. Disponible:http://scielo. es / scielo.php?script=sci_arttext\&pid=S0210$48062006000100010 \& \operatorname{lng}=\mathrm{es}$

11. Dugan E, Roberts C P, Cohen J, et al. Why older community-dwelling adults do not discuss urinary incontinence with their primary care physicians. J Am Geriatr Soc 2001; 49: 462-465.

12. Lose, G. The burden of stress urinary incontinence. Eur Urol Supplements 2005; 4: 5-10.

13. Petros, P.E., Ulmsten, U.I. An integral theory of female urinary incontinence. Experimental and clinical considerations. Acta Obstet Gynecol Scand Suppl 1990; 153: 7-31.

14. Franco A, Peri L, Alcaraz A. Incontinencia urinaria. Documento Asociación Española de Urología 2008. Disponible en: http:// www.aeu.es/Ultimas Publicaciones. aspx

15. Laurikainen E, Valpas A, Kivela A, Kalliola T, Rinne K, TakalaT, et al. Retropubic compared with transobturator tape placement in treatment of urinary incontinence: a randomized controlled trial. Obstet Gynecol 2007;109:4-11.

\section{Correspondencia:}

Alex Alberto Guibovich Mesinas

Dirección: Av. Alfonso Ugarte s/n, Lima, Perú.

Teléfono: 999653059

Correo electrónico: alexguibovichmesinas@yahoo.es 